\title{
Longitudinal study of lung function in a cohort of primary ciliary dyskinesia
}

\author{
A. Ellerman, H. Bisgaard
}

\begin{abstract}
Longitudinal study of lung function in a cohort of primary ciliary dyskinesia. A. Ellerman, H. Bisgaard. CERS Journals Ltd 1997.

ABSTRACT: Patients with primary ciliary dyskinesia (PCD) have pronounced stasis of their respiratory secretions and therefore recurrent lower airway infections, which raises concerns for the development of lung function.

Twenty four patients with PCD have been studied prospectively with a standardized regime in our clinic for 2-16 yrs with clinic visits, including spirometry 2-4 times per year, daily physiotherapy and monthly sputum cultures with subsequent specific antibiotic treatment.

Lung function was significantly lower in the 12 PCD patients entering the cohort as adults when compared to the PCD patients entering as children (forced vital capacity (FVC) 70 versus $85 \%$ predicted; forced expiratory volume in one second (FEV1) 59 versus $72 \%$ pred). The lung damage did not relate to the type of ciliary dyskinesia. During the subsequent surveillance of the groups for a median of 14 and $7 \mathrm{yrs}$, respectively, the lung function remained stable in most patients.

It is concluded that primary ciliary dyskinesia is accompanied by a progressive deterioration in lung function if undertreated, but lung function can be maintained with appropriate antibiotic treatment and regular physiotherapy. This emphasizes the need for early diagnosis of primary ciliary dyskinesia.
\end{abstract} Eur Respir J 1997; 10: 2376-2379.

\author{
Correspondence: H. Bisgaard \\ Pulmonary Service \\ Dept Paediatrics \\ Rigshospitalet \\ National University Hospital \\ Copenhagen \\ Denmark
}

Keywords: Lung function primary ciliary dyskinesia

Received: December 191996

Accepted after revision July 11997
Primary ciliary dyskinesia (PCD) is characterized by: chronic pulmonary symptoms including excessive phlegm, cough and recurrent infections, eventually causing a progressive deterioration in lung function; recurrent otitis media with secondary conductive hearing impairment; nasal polyposis; chronic sinusitis; chronic rhinorrhoea; and reduced male fertility. Defective dynein function in the sliding microtubules causes abnormal motility of cilia and sperm, which is the commonest cause of these clinical manifestations. In approximately half the patients, situs inversus is present [1]. The recurrent pulmonary infections are caused by the grossly impaired mucociliary transport in the respiratory tract causing stasis of the mucus within the bronchi. The diagnosis of PCD may be commonly delayed as the syndrome of bronchitis, sinusitis and otitis is easily mistaken for common infections. This may lead to many years of neglect and undertreatment of infections, which is a cause for concern because of the progressive lung damage.

To evaluate the prognosis of lung function in patients with PCD and the influence of a particular treatment regimen, we have studied a cohort of patients with PCD followed for 2-16 yrs in a prospective cohort study with a standardized regimen that consisted of regular clinic visits, physiotherapy and antibiotic treatment.

\section{Methods}

Rigshospitalet (National University Hospital) is a tertiary referral centre for paediatric pulmonology for the eastern half of Denmark. A cohort of PCD patients was initiated in the late 1970s, and the bronchopulmonary symptoms of part of this cohort have been reported previously [2]. By the end of 1994, 34 PCD patients of median age 21 yrs, (range 2-56 yrs) were attending our clinic. Eight of the patients, including two pairs of siblings, were born in the small, isolated community of the Faeroe Islands (approximately 30,000 inhabitants).

The diagnosis of PCD was based on the typical symptoms of bronchitis and recurrent sinusitis or otitis together with documented evidence of ciliary dysmotility. The cilia were taken from a scraping of the inferior nasal turbinate and the ciliary beat frequency was evaluated by microphoto-oscillography. The dyskinetic pattern was categorized as immotile, asynchronous or hyperfrequent [2-4]. Cystic fibrosis was excluded on the grounds of a normal sweat test.

Our standardized regimen for the PCD cohort consists of clinic visits every 3 months, where a clinical examination is performed together with spirometry. Every month a sputum culture is collected, preferably by expectoration, with nasopharyngeal suction as the last resort. Irrespective of symptoms, a 2 week course of antibiotic treatment is given if cultures show bacteria in relation to lower airway epithelium, and the corresponding bacteria are cultured. Antibiotic treatment is chosen from the sensitivity of the culture. If the same bacterial species is found repeatedly on more than two occasions, an extended course of 1-3 months of treatment is usually applied. Physiotherapy twice daily is carried out using 
the PEP (positive expiratory pressure) mask for a minimum of $10 \times 10$ breathings. The resistance at the outlet of the face mask is adjusted to generate a pressure within it of at least $10 \mathrm{cmH}_{2} \mathrm{O}$, which is checked at every clinic visit. In adult PCD patients, clinic visits are reduced to twice a year, but all patients are seen as needed in addition to the routine visits. Once a year, radiography of the chest is performed to monitor any development of bronchiectasis.

Forced spirometry (Spirotron; Dräger, Lübeck, Germany) using a noseclip was performed at every clinic visit guided by a trained nurse, and the best of three valid attempts was used as outcome. Published reference values for children [5], and compiled Danish reference values for adults were used.

The annual lung function is reported as the mean of the measurements performed at the clinic during the previous year. Nonparametric statistics were used for descriptive purposes as well as for between group comparisons (Mann-Whitney test).

\section{Results}

Twenty four patients of the PCD cohort had complied with the PCD regimen for a minimum of 2 yrs by the end of 1994 including regular spirometry during this period (table 1). Twelve of these were under the age of
18 yrs when they were enrolled into the PCD cohort control regimen. The children had been followed for a median of $7 \mathrm{yrs}$, and the adult group for a median of 14 years. Of the 10 patients excluded from this report, seven were excluded on the grounds that they were less than 6 yrs old and, thus, still too young to provide a 2 yr period of reliable lung function tests, the other three were $8-9$ yrs old and had only attended the clinic for less than $1 \mathrm{yr}$.

The forced vital capacity (FVC) ranged 30-104\% of predicted in adults and between $69-117 \%$ pred in children on entrance into the cohort (table 1). The FVC on entrance differed significantly between the two groups (median difference 17\%; 95\% confidence limits of median difference $3 \%-30 \%$ ), yet no clear correlation was seen between age and lung function within each of the two groups (table 1). The course of the FVC was stable in most patients after entrance to the PCD regimen (fig. $1 \mathrm{a}$ and $\mathrm{b}$; table 1). The patient with an FVC of $30 \%$ pred on entrance maintained it at that level for the following 15 yrs. This patient had chronic infection with Pseudomonas aeruginosa throughout the period, and was given 2 week $i . v$. courses with tobramycin and betalactam antibiotics on average twice a year over this period.

The forced expiratory volume in one second (FEV1) ranged 16-99\% pred in adults and $53-107 \%$ pred in children entering the cohort, also differing significantly

Table 1. - Patient characteristics and bacterial growth in sputum samples from lower airways obtained from the primary ciliray dyskinesia (PCD) cohort

\begin{tabular}{|c|c|c|c|c|c|c|c|c|c|c|c|c|}
\hline \multirow[t]{2}{*}{$\begin{array}{l}\text { Patient } \\
\text { No. }\end{array}$} & \multirow{2}{*}{$\begin{array}{c}\text { Age at } \\
\text { entrance } \\
\text { to cohort } \\
\text { yrs }\end{array}$} & \multirow{2}{*}{$\begin{array}{c}\text { Age by } \\
\text { end of } \\
1994 \\
\text { yrs }\end{array}$} & \multirow[t]{2}{*}{$\begin{array}{c}\text { Presence of } \\
\text { bronchiectasis }\end{array}$} & \multicolumn{2}{|c|}{$\begin{array}{l}\mathrm{FVC} \\
\% \text { pred }\end{array}$} & \multicolumn{2}{|c|}{$\begin{array}{l}\text { FEV1 } \\
\% \text { pred }\end{array}$} & \multirow[t]{2}{*}{$\begin{array}{c}\text { Cilia } \\
\text { function }\end{array}$} & \multirow{2}{*}{$\begin{array}{r}H i \\
\%\end{array}$} & \multirow[b]{2}{*}{ posit } & \multirow{2}{*}{$\begin{array}{l}\mathrm{Sa} \\
\text { cultul }\end{array}$} & \multirow{2}{*}{$\begin{array}{l}P a \\
\text { res }\end{array}$} \\
\hline & & & & $\begin{array}{l}1 \mathrm{st} \\
\text { year }\end{array}$ & $\begin{array}{l}\text { End } \\
1994\end{array}$ & $\begin{array}{l}1 \mathrm{st} \\
\text { year }\end{array}$ & $\begin{array}{l}\text { End } \\
1994\end{array}$ & & & & & \\
\hline \multicolumn{13}{|c|}{$\leq 18$ yrs of age at enrollment } \\
\hline 1 & 2 & 11 & - & 69 & 74 & 70 & 64 & A & 95 & 40 & 0 & 0 \\
\hline 2 & 4 & 13 & - & 87 & 85 & 88 & 74 & A & 83 & 27 & 0 & 0 \\
\hline 3 & 6 & 15 & - & 116 & 84 & 103 & 63 & A & 71 & 29 & 50 & 0 \\
\hline 4 & $6^{*}$ & 13 & - & 83 & 104 & 84 & 83 & A & 50 & 54 & 46 & 7 \\
\hline 5 & 7 & 9 & - & 117 & 114 & 107 & 93 & A & 44 & 29 & 0 & 0 \\
\hline 6 & $8^{*}$ & 17 & - & 85 & 93 & 65 & 67 & A & 92 & 23 & 8 & 0 \\
\hline 7 & 11 & 20 & + & 80 & 79 & 65 & 71 & I & 45 & 24 & 12 & 10 \\
\hline 8 & 11 & 26 & - & 93 & 120 & 53 & 64 & A & 52 & 16 & 55 & 0 \\
\hline 9 & 12 & 16 & - & 83 & 85 & 74 & 74 & A & 73 & 18 & 32 & 0 \\
\hline 10 & 13 & 25 & + & 77 & 79 & 60 & 59 & A & 39 & 23 & 23 & 13 \\
\hline 11 & 17 & 32 & - & 85 & 100 & 76 & 76 & A & 87 & 24 & 17 & 2 \\
\hline 12 & 18 & 34 & - & 87 & 79 & 69 & 64 & A & 73 & 3 & 35 & 1 \\
\hline Median & 9.5 & 16.5 & & 85 & 85 & 72 & 69 & & 72 & 24 & 20 & 0 \\
\hline \multicolumn{13}{|c|}{$>18$ yrs of age at enrollment } \\
\hline 13 & 22 & 26 & + & 90 & 87 & 81 & 73 & A & 50 & 7 & 43 & 0 \\
\hline 14 & 24 & 36 & & 80 & 76 & 49 & 60 & I & 20 & 20 & 16 & 27 \\
\hline 15 & 26 & 41 & - & 104 & 110 & 99 & 106 & $\mathrm{H}$ & 38 & 6 & 43 & 20 \\
\hline 16 & 28 & 38 & & 76 & 72 & 61 & 64 & A & 40 & 8 & 1 & 36 \\
\hline 17 & 29 & 43 & + & 74 & 82 & 79 & 86 & A & 71 & 20 & 5 & 0 \\
\hline 18 & 30 & 45 & + & 84 & 77 & 70 & 60 & I & 83 & 16 & 3 & 2 \\
\hline 19 & 30 & 42 & + & 63 & 78 & 57 & 45 & A & 63 & 19 & 18 & 7 \\
\hline 20 & 36 & 46 & - & 55 & 69 & 33 & 44 & A & 75 & 28 & 14 & 0 \\
\hline 21 & 36 & 41 & - & 66 & 61 & 26 & 28 & A & 84 & 34 & 13 & 0 \\
\hline 22 & 33 & 48 & + & 30 & 27 & 16 & 19 & A & 1 & 1 & 8 & 97 \\
\hline 23 & 43 & 56 & + & 55 & 79 & 55 & 64 & $\mathrm{H}$ & 83 & 19 & 0 & 2 \\
\hline 24 & 53 & 55 & - & 64 & 66 & 62 & 70 & I & 0 & 0 & 0 & 0 \\
\hline Median & 30 & 44 & & 70 & 76.5 & 59 & 62 & & 57 & 18 & 11 & 2 \\
\hline
\end{tabular}

*: pair of siblings with primary ciliary dyskinesia. $\dagger$ : detected by radiograph. FVC: forced vital capacity; FEV1: forced expiratory volume in one second; \% pred: percentage of predicted values; +: present; -: absent; A: asynchronous; I: immotile; H: hyperfrequent. Hi: Hemophilus influenzae; P: Pneumococcus; Sa: Staphylococcus aureus; Pa: Pseudomonas aeruginosa. 

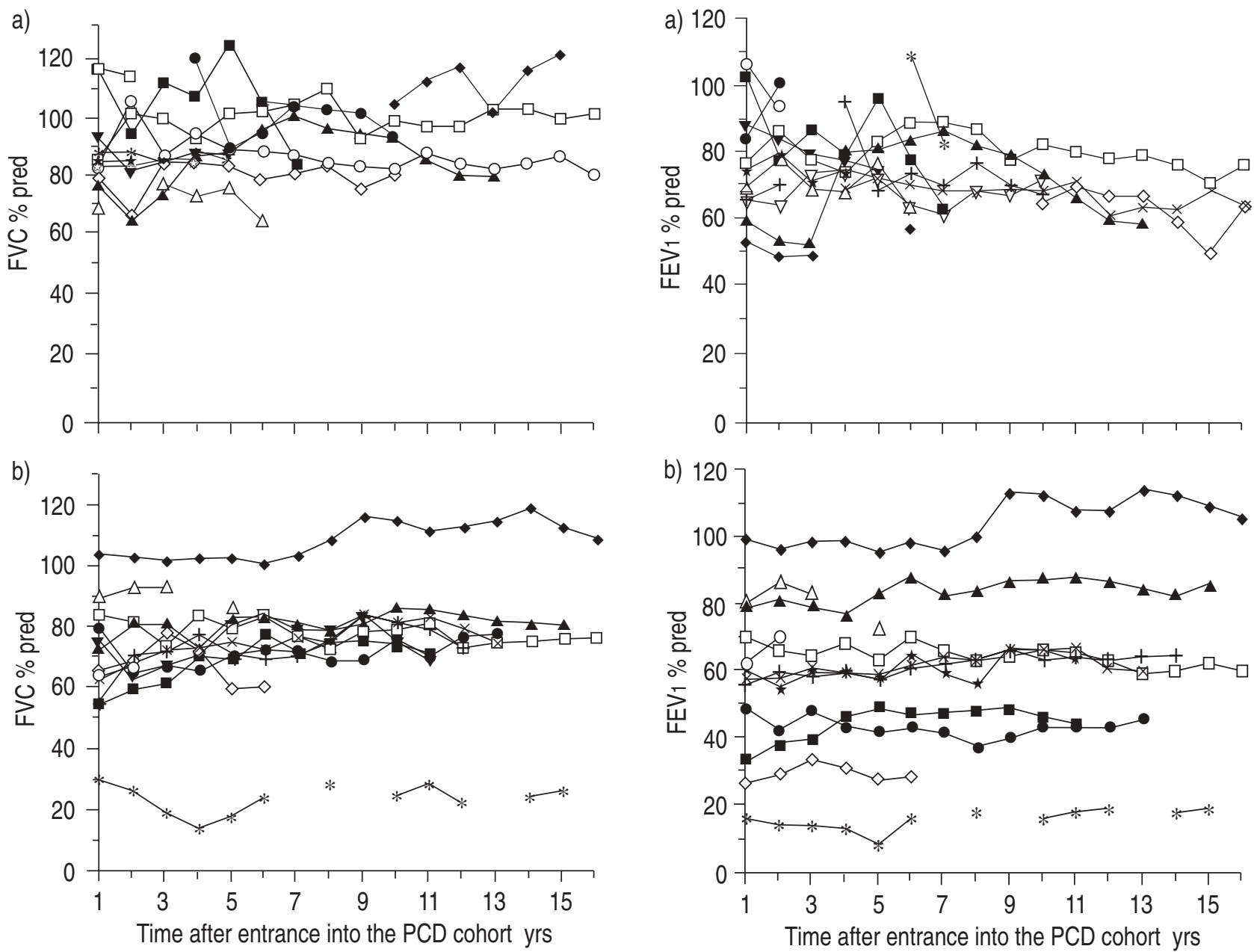

Fig. 1. - Forced vital capacity (FVC) \% predicted during the 2-16 yrs of observation in the group of pulmonary ciliary dyskinesia (PCD) patients entering the cohort in: a) childhood; and b) adulthood. Each symbol represents an individual patient.

(median difference 16\%; 95\% confidence limit of median difference $0-37 \%$ ) (table 1). The course of FEV1\% pred was stable during the observed period, though some patients exhibited a slight increase (fig $2 \mathrm{a}$ and $\mathrm{b}$; table $1)$. Lung damage was not related to the pattern of ciliary dyskinesia (table 1).

Bronchiectasis was found on chest radiograph in two patients from the child subgroup and six from the adult subgroup.

Bacterial findings were positive in $81 \%$ of sputum cultures in adults and in $83 \%$ in children, dominated by Hemophilus influenza, Pneumococcus, Staphyloccus aureus and rarely Pseudomonas aerurinosa with no differences between the two subgroups (table 1). The antibiotics used were mainly penicillins and erythromycin.

\section{Discussion}

On admittance to our clinic for a diagnosis of PCD, the FVC as well as the FEV1 was reduced in most of the 12 patients entering as adults and significantly more so as compared to the subgroup of 12 PCD patients entering as children. This suggests that lung function is damaged during the course of the disease. Spirometry

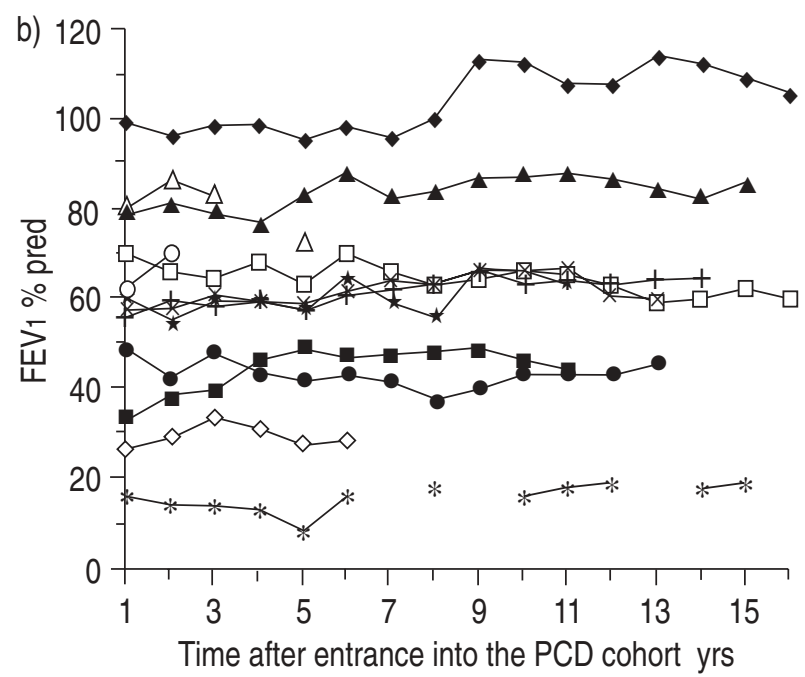

Fig. 2. - Forced expiratory volume in one second (FEV1) \% predicted during the $2-16$ yrs of observation in the group of pulmonary ciliary dyskinesia (PCD) patients entering the cohort in: a) childhood; and b) adulthood. Each symbol represents an individual patient.

was subsequently measured repeatedly two to four times per year, and represented as yearly mean values. The lung function did not deteriorate during the observation period of 2-16 yrs, which suggests that the regimen of physiotherapy and monthly sputum cultures with specific antibiotic treatment, irrespective of symptoms, prevents lung damage.

The standardized regimen did not change during the period. Only the antibiotics available seem to present a possible bias over the period, though this has probably had little effect as penicillins and erythromycin constituted the vast majority of antibiotic treatments.

These findings are in agreement with previous reports on lung function in PCD which found reduced lung function that stabilized after diagnosis and followup. CORKEY et al. [6] reported retrospectively on seven patients, 12-25 yrs, where lung function had been performed on at least three occasions within periods of 4 14 yrs. The FVC was within normal limits and the FEV1 below $80 \%$ pred and both remained stable during the observed period [6]. Kollberg et al. [7] reported on 14 PCD patients, 25-40 yrs of age, where the mean values for FVC and FEV1 \% pred were 76 and 59, respectively, but conducted no follow-up. These reports support the theory that lung damage occurs due to undertreatment 
of recurrent lung infections and appears to be prevented by accurate diagnosis and appropriate clinical care.

Bronchiectasis is a late complication of the recurrent infections, and was only revealed in eight of our 24 patients by chest radiograph. Computed tomography (CT) scan may reveal bronchiectasis at an earlier stage. However, lung function seems the most appropriate method for monitoring the disease, and the finding of bronchiectasis would have no effect on the management of the patient. Therefore, we do not expose our patients to CT scan as part of our routine management plan.

PCD is a rare disease with an estimated incidence of 1 in 15,000-30,000. It is inherited as an autosomal recessive disease. The genetic linkage is illustrated in the present cohort by three pairs of siblings with identical structural abnormalities, and an accumulated incidence in the remote isolated small community of the Faeroe Islands. Though such genetic linkage does occur, it is far from useful for clinical screening. Situs inversus is only apparent in about half the PCD patients and is, therefore, not exhaustive as a diagnostic criterion. The clinical suspicion has to rely on a syndrome of bronchitis with chronic upper airway secretions from ear and nose. Electron microscopy may reveal abnormal ultrastructure in the cilia, but ciliary dyskinesia with apparently normal ultrastructure has been reported [8-10]. The specific diagnosis relies on demonstration of inefficient ciliary function, by microscopy and photometric techniques [11], which is only available in very specialized centres. Such lack of specific clinical symptoms together with difficult access to diagnostic investigations are likely to cause considerable underdiagnosis, which raises concern for the outcome of lung function in undiagnosed patients. This concern is substantiated in the present study. It is the important conclusion of the present longitudinal cohort study, that progressive and significant lung damage occurs up to the time of diagnosis. In our cohort, lung function was stabilized thereafter, probably due to our intensive intervention, primarily with regular antibiotic courses against cultured bacteria, irrespective of the symptoms of the patient. Regular monthly sputum cultures are a central part of our regimen. Growth of $H$. influenza, pneumococci or $S$. aureus was found in approximately $80 \%$ of sputum cultures and with prevalence in that order. $P$. aeruginosa and other bacteria were rare. We did not routinely look for viral infections. Significant bacterial growth in these cultures were treated with 2 week courses of oral antibiotics as determined from the sensitivity of the bacteria. Accordingly, our patients were treated with oral antibiotics for approximately 20 weeks.year-1, in most cases with penicillins or erythromycin. This may seem an aggressive regimen and is unproven, though the present data seem to attest to its efficacy. A similar regimen has been applied in patients with cystic fibrosis, apparently with clinical success, though it has only been documented against historical controls [12].

The present findings illustrate the importance of early diagnosis of primary ciliary dyskinesia and intensive physiotherapy and antibiotic treatment. The defect is congenital and symptoms are present from early life, which stresses the importance for paediatricians to be aware of this disorder as a relevant albeit rare, differential diagnosis in children with recurrent symptoms from upper and lower airways. Provided such early diagnosis and intensive treatment is available, prognosis for maintaining the lung function seems good.

\section{References}

1. $\quad$ Sleigh MA. Primary ciliary dyskinesia. Lancet 1981 ; ii: 476.

2. Pedersen Stafanger G. Bronchopulmonary symptoms in primary ciliary dyskinesia. Eur J Respir Dis 1983; 64 (Suppl. 127): 118-128.

3. Pedersen M. Specific types of abnormal ciliary motility in Kartagener's syndrome and analogous respiratory disorders. Eur J Respir Dis 1983; 64 (Suppl. 127): 78-90.

4. Pedersen M, Nielsen MH. Abnormal ciliary motility as a cause of chronic airway disease. In: Götz, Stur: Pediatric Disease. Prog Resp Res 1981; 17: 190-196.

5. Rosenthal M, Bain SH, Cramer D, et al. Lung function in white children aged 4-19 years: I - Spirometry. Thorax 1993; 48: 794-802.

6. Corkey CWB, Levison H, Turner JAP. The immotile cilia syndrome. Am Rev Respir Dis 1981; 124: 544-589.

7. Kolberg H, Mossberg B, Afzelius BA, Philipson K, Camner P. Cystic fibrosis compared with the immotile cilia syndrome. Scand J Respir Dis 1978; 59: 297-306.

8. Greenstone M, Dewar A, Cole PJ. Ciliary dyskinesia with normal ultrastructure. Thorax 1983; 38: 875-876.

9. Greenstone M, Rutman A, Pavia D, Lawrence D, Cole PJ. Normal axonemal structure and function in Kartagener's syndrome: an inexplicable paradox. Thorax 1985; 40: 956-957.

10. Escudier E, Escalier D, Homasson JP, Pinchon MC, Bernaudin JF. Unexpectedly normal cilia and spermatozoa in an infertile man with Kartagener's syndrome. Eur J Respir Dis 1987; 70: 180-186.

11. Greenstone M, Cole PJ. Primary ciliary dyskinesia. Arch Dis Childhood 1984; 59: 704-706.

12. Frederiksen B, Lanng S, Koch C, Høiby N. Improved survival in Danish center-treated cystic fibrosis patients: results of aggressive treatment. Pediatr Pulmonol 1996; 21: $153-158$. 